\title{
Workshop focuses on the rise in MSE undergraduates
}

\author{
By Susan B. Sinnott, David F. Bahr, Angus Rockett, Michael D. Williams, and Hatem Zurob
}

$\mathrm{I}^{\mathrm{s}}$ n September 2019, a US National Science Foundation (NSF)-supported workshop, "The Enrollment Floodgates Are Open-Best Practices in Materials Science and Engineering Undergraduate Education for Rising Enrollments," was held at The Pennsylvania State University. In attendance were representatives in leadership positions from universities, a variety of companies, national laboratories, and government organizations. This group (Figure 1) met over three days to discuss the steady and significant rise in undergraduate students graduating with degrees in materials science and engineering (MSE) over the last decade as a result of rising enrollments in many existing MSE programs and the creation of new undergraduate programs. The workshop attendees discussed the impact of these changes on the educational mission and opportunities for employment and the establishment of best practices for the future.

\section{Trends and key drivers}

The trend in enrollment in materials programs since the mid-2000s generally has been one of consistent increase; however, in most individual programs, enrollment has gradually been leveling off since 2016. Change in enrollment in programs in the United States from 2016 to 2018 include an even distribution of programs increasing and decreasing in size, and the changes are not correlated with the size of the program (see Figure 2). Some of the largest programs are growing by more than $10 \%$, and others are shrinking by similar amounts. Smaller programs display a similar behavior. There seems to be little consistency in the types of programs that behave in one way or another, with rising and falling enrollments spread among large and small programs in R1 and R2 type (research-oriented with very high or high levels of activity) institutions. Similar trends are also seen in enrollments in Canada.

Several factors have been driving increases in program sizes in materials, even while anecdotal evidence indicates that lack of awareness within the general public of MSE as a field unto itself continues to hold it back. Primary drivers for growth include:

(1) Public institutions, in particular, have seen continuous decreases in state funding support. Increases in tuition have not kept pace with these decreases. Only tuition combined with economies of scale can manage the pressures of economic forces. Nearly all public and most private engineering programs, therefore, have increased enrollment overall. As a result, popular and well-recognized programs, such as mechanical engineering, have been overloaded and have

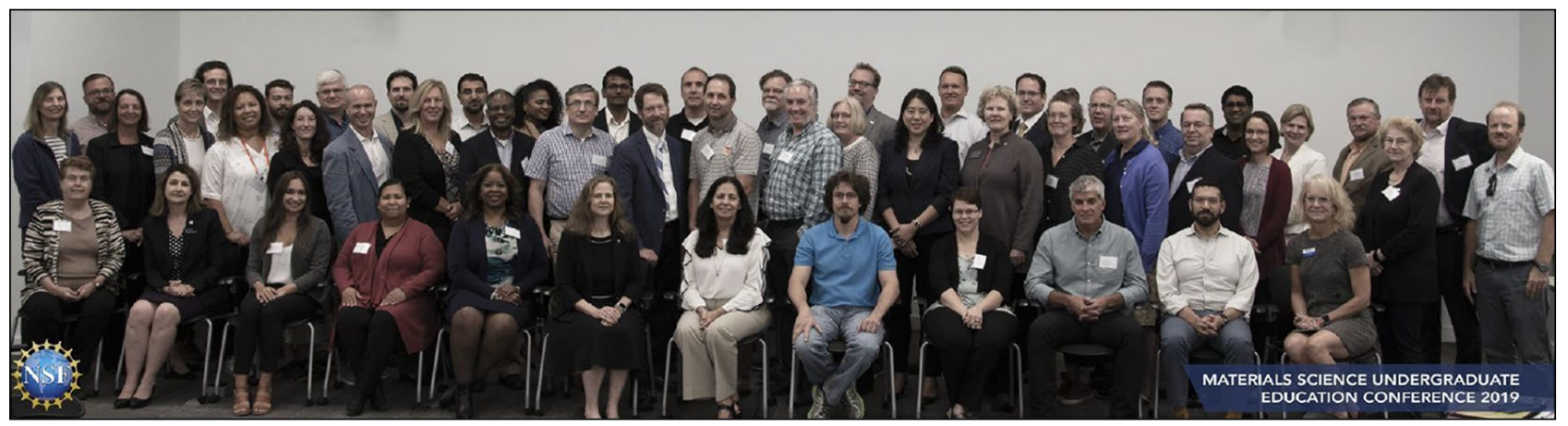

Figure 1. Attendees of the workshop titled "The Enrollment Floodgates Are Open-Best Practices in Materials Science and Engineering Undergraduate Education for Rising Enrollments," which was held on the University Park campus of The Pennsylvania State University, September 9-11, 2019. 
adopted policies that encourage off-loading of enrollment to smaller programs. Initial dramatic increases in enrollment in materials programs occurred at institutions where enrollments were controlled or explicitly capped. As time has progressed, these materials programs have seen enrollments stabilize at near double where they were 10-20 years ago. Programs at institutions where enrollment is not capped in wellknown disciplines took longer to grow but are following the same trend as the schools with caps. This is driven, in part, by indirect efforts to redirect students and because of the inherent popularity of materials programs.

(2) The materials field is well known in the research community, having contributed in critical ways to many technologies. This has resulted in departments across engineering colleges hiring materials faculty, even while MSE is not a well-known field among high school students and undergraduates. Nonetheless, the experience of students in the field is spreading its reputation for providing good career opportunities. It has a reputation within its student body for engaging hands-on programs with strong laboratory components. The potential for loss of this connection is one of the most significant challenges when enrollments grow. The traditionally smaller class sizes compared to those in well-known programs promotes effective faculty-student interactions and is attractive to students, especially to women and other under-represented groups, even as evidence indicates that the correlation between class size and student success is subtle. ${ }^{1}$ Research indicates that under-represented groups benefit the most from a close engagement with advisers and mentors. ${ }^{2}$ At the same time, increasing enrollments place

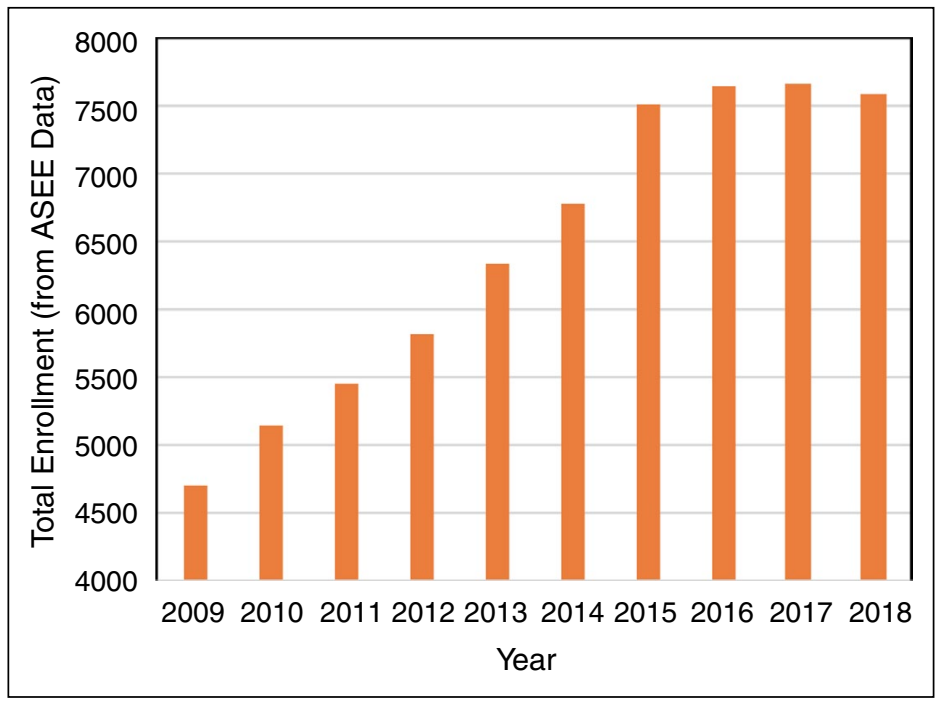

Figure 2. Total enrollment reported to the American Society for Engineering Education (ASEE) for the past 10 years showing a continuous increase until 2016. While the total enrollment has risen, individual programs show rises and falls at various times during the period reflected in the data.

increasing pressure on the laboratory component. It is straightforward to increase enrollments in lecture classes, but laboratories are more difficult to expand.

(3) Several new undergraduate programs are being developed across the United States, including at the University of Virginia, the University of Buffalo, the University of Delaware, and Texas A\&M University. This means that even though some MSE programs, such as those at selective private institutions, have controlled, relatively small, and flat enrollment numbers, the total number of undergraduate students in North America being educated in the field has substantially increased, and this trend is expected to continue to increase in the future.

\section{Recruiting and helping students find their academic major}

A significant and continuing issue with managing enrollments in MSE programs is awareness of the field. Various approaches have been used to address this issue with success, including holding summer camps for high school teachers, many sponsored by the ASM International Foundation, and engaging in outreach visits to high schools. Summer camps have an advantage in that they both promote awareness and provide effective demonstrations and background for teachers so that they can provide introductions to materials in their science classes. Outreach visits to regional high schools have been found to be effective using current undergraduate students as ambassadors, and participants shared that this is particularly effective when the students are from the high school being visited. On-campus summer programs and design competitions for high school and middle school students are effective and have been implemented in both the United States and Canada. Finally, it was noted that parents are highly influential in the choices students make, so contact with the parents is important. This can be accomplished through participation in community events, such as science fairs. It is important for materials programs to collaborate in development of outreach and publicity and to share best practices, such that these efforts are broadly applied across the globe. 


\section{Impact on diversity, inclusion, and access}

Diversity, inclusion, and access are deeply embedded into all that we do at the student and faculty levels. Some MSE programs participating in the workshop have performed well with respect to participation and inclusion of women, but minority participation is lacking, particularly for African Americans. The percentage of African Americans obtaining BS degrees in physical sciences and engineering grew by $43 \%$ from 2005 to $2015 .^{3}$ This is significant when compared to the fact that the overall increase in the number of BS degrees obtained in the United States was 32\% for this same period. Unfortunately, this flood of BS degrees is not maintained for African Americans in science, technology, engineering, and math (STEM). While the overall growth in STEM is $55 \%$, that for African Americans is substantially lower at $36 \%$. This is not enough to match the demographic growth of underrepresented minorities in the United States. The discussion at the workshop and some online data diving on the part of some participants found that the losses in STEM are offset by gains in other areas such as business and medicine. The Pew Research Data on demographic trends provided by Acoff for the Underrepresented Minority Panel suggests that the Caucasian population will cease to have its numerical advantage by 2050 , when it will be exceeded by the total number of minorities (primarily Hispanics and African Americans). ${ }^{4}$ This shift in the majority status will portend a steep decline in the number of US nationals pursuing careers in STEM. This is seriously concerning in scope, but it also provides a unique opportunity for women and minorities to fill the urgent need for materials scientists and engineers.

To this latter point, a recurring theme among workshop participants was that students largely are initially not aware of MSE until they take introductory general engineering courses. Many programs, such as the undergraduate Penn State Materials Science and Engineering program, have taken advantage of this phenomenon to recruit students who start in other majors. ${ }^{5}$ Additionally, students are driven from other engineering and physical science disciplines because of enrollment caps, minimum GPA requirements, and lack of inclusion. The consensus is that a targeted approach must continue to be taken to educate, raise awareness of the field, and involve women and minorities at all points in the pipeline. Suggestions raised included starting the mentoring process with middle school students and collaborating with minority STEM organizations to leverage participation. These organizations include the Society of Women Engineers, National Society of Black Physicists, National Society of Hispanic Physicists, National Society of Black Engineers, National Organization for the Professional Advancement of Black Chemists and Chemical Engineers, and National Action Council for Minorities in Engineering. Additional items that are recommended are partnering with federal programs such as the Louis Stokes Alliances for Minority Participation (LSAMP) program, the NSF Improving Undergraduate STEM Education: Hispanic-Serving Institutions (HIS Program), and the NSF Alliances for Graduate Education and the Professoriate (AGEP), which are actively introducing students into the STEM pipeline and developing inclusive activities at the program/ department/school level. Partnering with Historically Black Colleges and Universities (HBCUs) in dual degree programs, where students spend three years at the HBCU and then pipeline directly into MSE programs, students spend two years to complete both the HBCU degree and the MSE degree, and hosting research experience for undergraduates (REUs) targeted at minorities and women will lead to increases in diversity.

With respect to admission, removing enrollment caps and decreasing the emphasis on standardized exams or GPAs for entrance criteria while taking a holistic view of admission and addressing unintentional bias are emphasized. A short-term effect of removing enrollment caps in other majors might be a decrease in MSE enrollments. Given that MSE is a discovery major and enrollment caps in popular programs such as mechanical engineering have allowed some MSE programs to double in size, this growth comes with a risk. When the enrollment caps were relaxed at McMaster University, for example, student enrollment in the MSE program dropped by $50 \%$ in one year. It took three years, and many new outreach initiatives, to recover the original student numbers. It is therefore recommended that programs dedicate additional resources for outreach to coincide with removing enrollment caps. Utilizing dedicated recruiters and leveraging social media are recommended practices. Programs are encouraged to also support educational podcasts, such as "Dope Labs" (https ://www.dopelabspodcast.com/).

Some of the best practices for building and maintaining a program that is strong in diversity, inclusion, and access include the mentoring of students, which is important to retain minority undergraduate students in STEM disciplines. ${ }^{2}$ Undergraduate research is also important for retention and encouraging interest in graduate school. Outreach and engagement with students in high school and middle school is critical with respect to attraction to STEM. Importantly, role models are key to encourage students from underrepresented groups to pursue careers in STEM fields such as MSE.

\section{Employment opportunities}

The workshop included panels to provide the perspective of employers of primarily undergraduate MSE programs, including industry, national laboratories, and graduate programs in MSE. Results of a similar survey were published recently in the Journal of Metals. ${ }^{6}$ 


\section{Industry}

The message from the industrial representatives at the workshop was that there is a need for graduates in the materials space with specialized expertise in areas such as metallurgy, polymers, glass, aerospace materials, and energy storage at all degree levels. While many of these areas of expertise are not new, the industrial representatives indicated that necessary technical expertise that is required is often different from the expertise required 10-15 years ago. For instance, today there is a need for expertise in extreme environments, particle impacts on materials at very low temperatures, and a variety of materials for aerospace applications. In general, there is a need for new hires to understand how equipment works and how things are made. In contrast, hires at the MS or $\mathrm{PhD}$ levels are not necessarily trained for solving complex problems but instead are focused on project management. The industrial panel stressed that increased exposure to computational methods is very important for students, as today's industrial setting requires working with lots of data analytics. A recommended practice to emerge from the discussion was that students be exposed to computational methods to encourage them to think in different directions, including being able to analyze data sets and draw conclusions.

The expertise valued by industry ${ }^{7}$ among MSE degree holders include hands-on skills, especially in making materials, problem-solving skills, and technical curiosity. In addition, the ability to work as part of a team, including cross-functional teams, and coordinating across multiple departments is valued. Process engineering capabilities are also important, including quality, statistics, and design of experiments. Practical experiences, including internships and industrial relationships, are highly valued, as are leadership skills, with an emphasis on project management in terms of milestones, schedule, budget, and deliverables.

The industrial panel emphasized that communication skills are key, especially being able to communicate with formal presentations and in informal settings with a variety of people, such as workers on a plant floor, engineers, supervisors, and executives. For example, being able to communicate with operators is necessary to effectively obtain critical information and ultimately improve the ability of operators to do their job. This is aided by prior experience working with other engineering disciplines. New graduates need to be able to articulate a technical accomplishment and its impact. The panel noted the impact of generational differences in communication in an industrial setting, with some generations preferring email and others preferring instant messaging, and they emphasized that electronic written communication is not a good substitute for in-person communication either face-toface or on the phone or through a video call.

From a practical standpoint, operational experience is more important than more knowledge learned in courses. There was discussion about the costs and benefits of industrial internships and whether these experiences should be increased in undergraduate curricula. All agreed that internships require a significant amount of effort for industrial hosts to be successful, but they are important. For some companies, internships are used to determine if they will hire the person by determining if they fit in the corporate culture (projects and presentations). In other cases, internships lead to jobs as contractors that may last over multiple years. For other companies, there is concern that requiring an internship would negatively affect the quality of new hires. In these cases, internships are used as trial periods or a three-month job interview. There is a big difference between quality internships and those with lesser impact, and interns tend to share this information with peers. Longer internships would allow for a more in-depth experience for students and be more meaningful in the case of fundamental research; however, it would be challenging to identify enough industrial staff to enable that level of educational engagement. In some cases, a mentorship model might be better. Training at a national laboratory is also viewed as valuable for a future position in industry.

Some different avenues to achieve desired attributes in industry for graduates with BS degrees in MSE include an engineering-focused MBA to incorporate building other skills. In addition, skills can be built into senior engineering design or in other core courses within the undergraduate curriculum. It is also effective to encourage interactions with elective and/or invited speakers to incorporate professional skills. Other ideas included increasing the scope/length of capstone projects and working with more interdisciplinary/ cross-functional teams, as well as more specialization in undergraduate programs (e.g., metallurgy and polymers) and a variety in electives in combination with undergrad research.

It is recommended that curricula weave the same skill set, such as project management, through multiple classes to strengthen students' capabilities. In some engineering disciplines, such as civil engineering, it is recommended that graduates have an MS degree before they can fully practice in their field. Funding senior capstone design projects by industry is a way to interview a team of students at the same time and evaluate how well they work on a team; this approach is ultimately less expensive than a traditional internship and a more effective implicit interview, although not a good alternative to an internship. Industrially supported capstone design projects are valuable experiences for the students on the team and are a good way for companies to stay connected to a university, but it still takes resources. Projects in areas with proprietary potential are especially challenging.

Given that internship opportunities are limited and typically filled with the best undergraduate students, some 
attention is needed for students who are not at the top of their class who would benefit from additional formal education. At the same time, additional coursework may be less necessary than making sure that existing courses instill passion and drive in the students.

\section{National laboratories and organizations}

The panel of members from national laboratories and organizations indicated that there is fundamental interest in hiring in the MSE discipline across all fields, as knowledge of materials is necessary for fundamental research to manufacturing and systems support. In general, there is low turnover, but a substantial fraction of scientists and engineers at laboratories are within 10 years of retirement, so demand is expected to keep increasing during the next decade as labs continue to grow.

Unlike with industry, recruiting generally takes place through a research collaboration network and not through on-campus recruiting. Networking at conferences and one-on-one interactions with professors and their students aid in the hiring process. Frequently, graduate students are hired at the postdoctoral level, which allows for indepth evaluation before consideration for a long-term position. Recruiting can also take place via summer programs, where faculty and graduate or undergraduate students work together in the lab. There are also opportunities for graduate students to work at labs in specialized programs. National laboratories work with undergraduate students, and these opportunities tend to be cyclic through programs such as undergraduate research fellow programs, the Reserve Officer Training Corps (ROTC) program, Oak Ridge Institute for Science and Education (ORISE), and coops. In some cases, members of the lab are actively engaged in STEM outreach at local middle and high schools as well as in outreach to the public. Strategic centers of excellence at universities are a valued mechanism to diversify the workforce in national labs.
These labs are looking for a workforce that has a strong technical background. In the case of openings in materials science, there is often interest in hiring those with expertise in related areas such as biology, chemistry, artificial intelligence, and computational methods. Other expertise that is highly valued includes critical thinking skills, interdisciplinary knowledge, the ability to work on teams, and the drive and interest to collaborate and learn from others. In addition, good communication and writing skills are desirable. Clearances are an issue that can affect new hires, especially those who are not US citizens.

\section{Graduate schools}

Graduate schools want and need more undergraduate students with a materials focus. However, graduate schools are competing with other employers for these students, with an increasing number of students choosing to go into industry. The US Bureau of Labor Statistics indicates that MSE employment rose until 2015, where it leveled off after a recession dip. The number of undergraduate students in MSE programs who choose to go to graduate school has dropped over the last decade. For example, at the University of Maryland, in 2013, approximately $50 \%$ of undergraduate students went on to graduate school and approximately 50\% went on to MSE-related industry. In contrast, in 2018, approximately $33 \%$ of undergraduate students went to MSE-related industry, 33\% went to other types of industry, such as consulting, and $33 \%$ went to graduate school. Similar trends have been observed in other programs, with the net result showing a smaller fraction of MSE undergraduate students from US programs going to graduate school in engineering. Students who carry out research as undergraduates are more likely to go on to graduate school, while those who gain early experience in industry, through internships and co-ops, are most likely to work in industry after graduation.
The number of MSE programs offering the MS degree increased from 65 to 75 institutions between 2013 and 2018 . Interestingly, for every five BS degrees in MSE, two MS degrees are awarded. One important driver is the income generated for MSE programs that offer these degrees, especially MS degrees that do not require a thesis. The largest MSE MS programs in the United States are at the University of Southern California, the University of Florida, Stanford University, the University of California-San Diego, and the University of Pennsylvania. The traits exhibited in successful MS programs vary by institution, and, in general, programs that are adaptable, flexible, and align well with the strengths of the home department, college, or university as a whole are most effective.

At the University of Florida, there was an $85 \%$ growth in MSE MS degrees since 2007, although the number of students enrolled has dropped by more than $66 \%$ since 2015 . Approximately $39 \%$ of the students enrolled in the program are international, although this percentage has dropped because of difficulties with visas to study in the United States and increasing global competition for outstanding graduate students in STEM disciplines. The University of Florida addressed the decline with targeted digital marketing, including social media and focused working groups. It also increased emphasis on experiential learning by reducing roadblocks for research experiences, adding graduate laboratory courses, creating customized MS degree tracks and certificates, and increasing $\mathrm{PhD}$ enrollment. Online options are also available to international students if there are visa issues.

\section{Recommended best practices for managing large enrollments}

Approaches to managing enrollment increases in undergraduate (and in some cases graduate) instructional laboratory courses include switching from running labs in series to running them in parallel (e.g., rotating students through labs in different order, allowing each 
lab to run concurrently with a portion of the enrolled students). This has the advantage that it can be scaled up and can handle many students easily without the need for many copies of each apparatus. The disadvantages are that it requires lab spaces that can accommodate the parallel sessions, and the labs cannot easily be coordinated with lectures in related courses.

Other approaches include offering more lab sections by making use of evenings and weekends. While not popular with either students or faculty/staff, this has the advantage of not requiring significant additional apparatus or space and allowing close coordination of laboratory and lecture topics. Laboratories also can be offered during summer sessions or other times when classes are not normally in session. This makes instrument usage more effective but suffers from the inability to coordinate with lecture topics in other courses, which typically would be offered during a regular semester. It also limits the ability of students to participate in summer internships, leads to difficulties in prioritizing enrollment during normal semesters (i.e., determining who will have to attend for summer), and summer sessions require additional tuition payments without a related benefit. Use of summer sessions would be most effective if integrated into the full regular curriculum where the related topics are offered at the same time or that are used regularly to enable participation in a co-op during the academic year.

Examples were discussed relative to fields (e.g., petroleum engineering) that experience large swings in enrollment, which make managing resources and staffing difficult. When enrollments are high, facilities must be expanded, and there is pressure to hire additional faculty, while lean enrollments reverse these trends. Therefore, it is very helpful to cap enrollments. Based on the evidence discussed at the workshop, enrollment controls have the advantage of being able to balance load from year to year and to plan for growth. This also allows for a greater number of admissions if extra students can be directed to under-enrolled programs without it negatively affecting the students. In any event, a significant increase in enrollment should not be expected to be accompanied by increases in faculty, although this may occur at institutions for other reasons. Pedagogy is important in courses, particularly instructional laboratories, and capstone senior design courses.

Some best practices can be shared with respect to incorporation of soft skills into curricula without adding new courses. These include emphasizing business practices, writing, and communication skills in existing courses and offering minors in strategic areas in cooperation with other departments or colleges. There are several models with respect to ensuring adequate hands-on experience, including the Materials Innovation and Learning Laboratory at the Georgia Institute of Technology, Maker Space at Texas A\&M University, and the Learning Factory at Penn State. In addition, undergraduate research and senior thesis opportunities, along with industrial co-ops and internships, provide hands-on laboratory or computational expertise, build critical thinking skills, and give experience working as part of an interdisciplinary team. Involvement with professional societies and technical organizations, including student chapters of the Materials Research Society and Material Advantage, are also key to broadening students' perspective and understanding of the field.

\section{Conclusions and future outlook}

It was clear at the time of this workshop that MSE programs were not yet at saturation. The impact of COVID-19 is still evolving at the time of this writing and is not yet fully apparent. Nonetheless, some things are coming into focus.

The transition to online learning during the COVID-19 crisis provided valuable insights on aspects of the curriculum that could be effectively delivered online. Content developed during the crisis could be used in the future to ease space limitations at rapidly expanding programs. Interactive online tutorials and computer labs (e.g., CAD, Materials Selection) could accommodate larger class sizes, which would otherwise be difficult to accommodate inperson, using the existing classrooms or computer labs. Remote instruction is also enabling software tools to engage a range of students in different ways. ${ }^{8}$ Several original approaches that involve creating emulators of laboratory equipment have also been developed or suggested. Examples of these include emulators of mechanical testing frames and of differential scanning calorimeters. Aside from the steps that involve inserting and removing the samples, these emulators function in the same way that the physical instrument operates. In addition to providing more flexibility in dealing with large class sizes, these new online tools could complement the content offered during in-person laboratories and provide students with opportunities to explore a material's response under various what-if scenarios.

Given the multidisciplinary nature of materials science, future growth of MSE programs will, to some extent, depend on major trends within the broader fields of physical science and engineering. For example, in looking back over the last 30 years, MSE programs experienced growth in parallel with the nanotechnology revolution, which created a need for graduates who understand the processing, structure, and property relationships of nanomaterials. More recently, the expansion of biomedical sciences brought about increased interest in biomaterials. The key question for MSE programs is to anticipate the next revolution that could potentially lead to additional demand for MSE graduates and significant expansion in MSE programs. Given the unprecedented growth in the field of computer science, several programs are presently preparing to offer degree options that combine materials engineering and computer science/ data analytics/machine learning. Some of the options being explored include a minor in computer science, as well as five-year programs that lead to a joint bachelor's degree in computer science 
and MSE. The manufacturing industry has expressed a need for graduates who understand engineering fundamentals as well as computer science. Efforts to fill this gap by recruiting computer scientists to work within manufacturing have had limited success because of the competition with other computer science careers. In addition to meeting the needs of industry, the training of materials engineers with strong expertise in computer and data science has the potential to revolutionize MSE fields, as it would accelerate the adoption of high throughput experiments, autonomous platforms, and machine learning within the field.

\section{Acknowledgments}

We gratefully acknowledge the support of NSF (DMR-1842175). We also acknowledge the participants of the workshop who contributed so greatly to its success. Special thanks to R. Allen Kimel and Lynnette D. Madsen for many helpful discussions.

\section{References}

1. C. Flaherty, "Much Ado About Class Size," Inside Higher Ed(June 18, 2020), https://www.inside highered.com/ news/2020/06/18/study-some-things-matter-moreclass-size-when-it-comes-student-success.

2. T. Feder, Phys. Today 72(10), 24 (2019).

3. L. Merner, J. Tyler, "Focus on Report: AfricanAmerican Participation Among Bachelors in the Physical Science and Engineering," (2019), https:// www.aip.org/statistics/reports/african-americanparticipation-among-bachelors-physical-sciences.

4. J.S. Passel, D. Cohn, US Population Projections: 2005-2050 (Pew Research Center, Washington, DC, 2008).

5. R.A. Kimel, S.B. Sinnott, MRS Bull. 43(4), 257 (2018).

6. S.E. Hunyadi, A. Singh, K. Clarke, "Workforce Development Survey Results: Industry, Government Laboratories, Academia, and Recent Graduates," JOM 72(10), 3312 (2020).

7. L.D. Madsen, E.B. Svedberg (eds.), Materials Research for Manufacturing: An Industrial Perspective of Turning Materials into New Products (Springer Series in Materials Science, Springer, New York, 2016).

8. D. Lederman, "What Do We Know About This Spring's Remote Learning?" Inside Higher Ed (June 10, 2020), https://www.insidehighered. com/digital-learning/article/2020/06/10/whatdo-we-know-and-what-should-we-try-learn -about-springs.

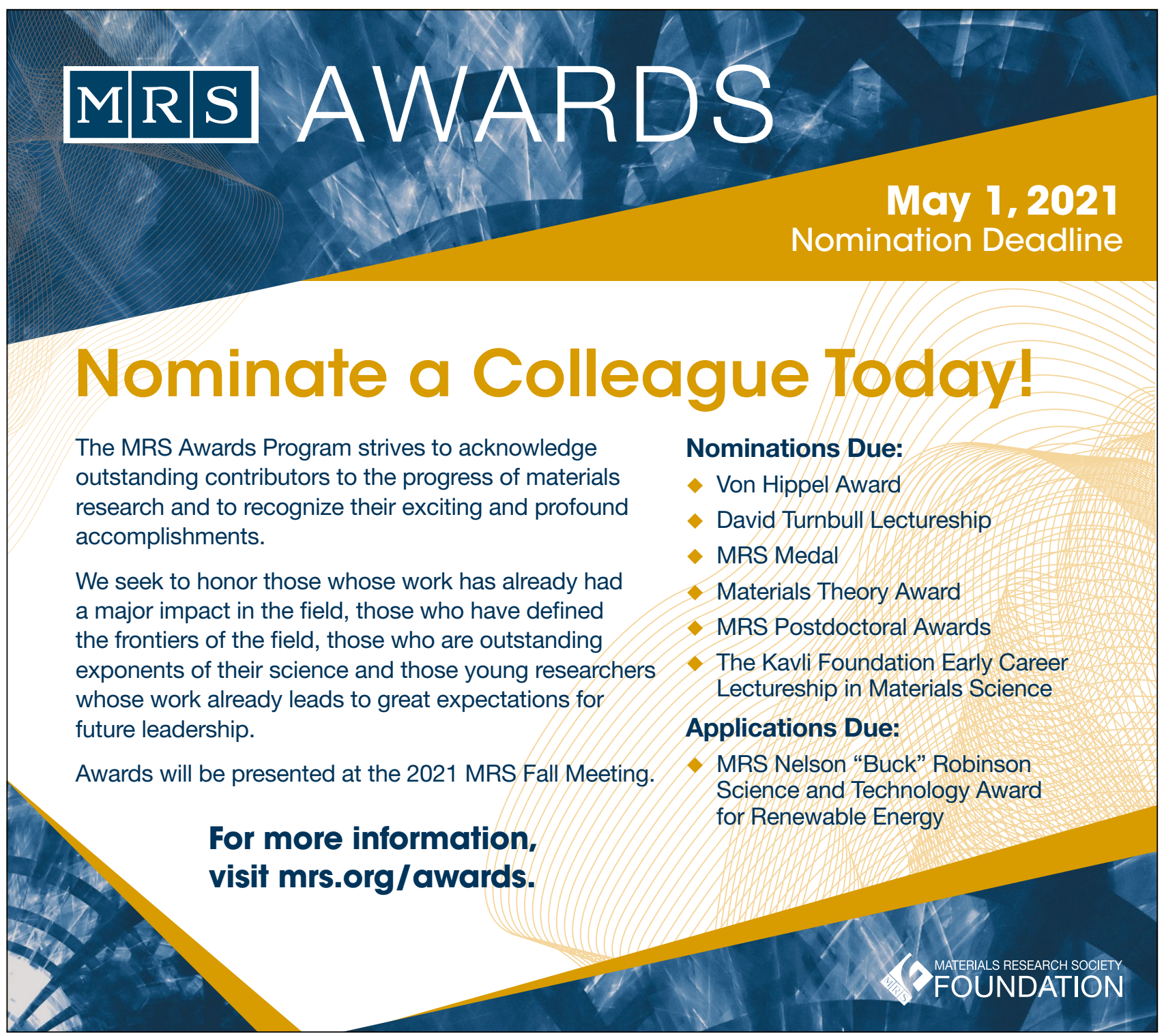

